\title{
The Bi-Elliptical Deformable Contour and Its Application to Automated Tongue Segmentation in Chinese Medicine
}

\author{
Bo Pang, David Zhang*, Senior Member, IEEE, and Kuanquan Wang, Member, IEEE
}

\begin{abstract}
Automated tongue image segmentation, in Chinese medicine, is difficult due to two special factors: 1) there are many pathological details on the surface of the tongue, which have a large influence on edge extraction; 2) the shapes of the tongue bodies captured from various persons (with different diseases) are quite different, so they are impossible to describe properly using a predefined deformable template. To address these problems, in this paper, we propose an original technique that is based on a combination of a bi-elliptical deformable template (BEDT) and an active contour model, namely the bi-elliptical deformable contour (BEDC). The BEDT captures gross shape features by using the steepest decent method on its energy function in the parameter space. The BEDC is derived from the BEDT by substituting template forces for classical internal forces, and can deform to fit local details. Our algorithm features fully automatic interpretation of tongue images and a consistent combination of global and local controls via the template force. We apply the BEDC to a large set of clinical tongue images and present experimental results.
\end{abstract}

Index Terms-Chinese medicine, deformable templates, medical biometrics, snakes, tongue image segmentation.

\section{INTRODUCTION}

\section{A. Automatic Tongue Diagnosis System}

$\mathbf{T}$ RADITIONAL CHINESE MEDICINE (TCM) has a history of thousands of years and its practitioners have accumulated very rich practical experiences in diagnostic methods. Tongue diagnosis [13] is one of the most important and widely used diagnostic methods in Chinese medicine. This method is also very valuable in clinical applications and self-diagnosis. The beauty of tongue diagnosis lies in its simplicity and immediacy. Whenever there is a complex disorder full of contradictions, examination of the tongue can often quickly clarify the main pathological processes. The color of the tongue body almost always reflects the true condition of the patient, especially in terms of the eight principles that are used in the identification of patterns. In addition, the tongue's appearance is a most useful gauge for monitoring the improvement or deterioration

Manuscript received September 1, 2004; revised April 4, 2005. This work was supported in part by the National Natural Science Foundation (NSFC), by the High-Tech 863 Fund, and by the Central Fund, Hong Kong Polytechnic University. The Associate Editor responsible for coordinating the review of this paper and recommending its publication was J. Duncan. Asterisk indicates corresponding author.

B. Pang and K. Wang are with the Department of Computer Science and Engineering, Harbin Institute of Technology, Harbin 150001, China (e-mail: bpang@hit.edu.cn; wangkq@hit.edu.cn).

*D. Zhang is with the Department of Computing, Hong Kong Polytechnic University, Kowloon, Hong Kong (e-mail: csdzhang@comp.polyu.edu.hk).

Digital Object Identifier 10.1109/TMI.2005.850552

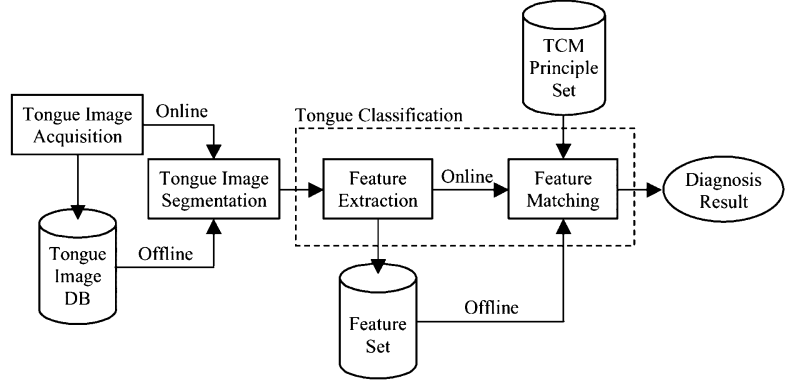

Fig. 1. Flowchart of a biometrics-based automatic tongue diagnosis system in TCM.

of a patient's condition. Moreover, tongue diagnosis is one of a few existing diagnostic techniques that are in accord with the most promising diagnostic directions pursued in the 21 st century: no pain and no injury.

Nowadays, increasingly powerful computer processors have made it possible to develop an automated tongue diagnosis system that is based on image processing and pattern recognition. In addition, biometrics, as a technology that uses human being's unique features to identify or verify persons [15], can be conveniently used in tongue diagnosis provided the tongue's appearance is viewed as a physical or behavioral feature and the patient's corresponding disorder is regarded as the "identity." Therefore, we introduce modern biometrics techniques to traditional tongue diagnosis and develop a biometrics-based automatic tongue diagnosis system, as illustrated in Fig. 1.

\section{B. Difficulties in Automatic Tongue Segmentation}

Tongue images can be captured by using a specific set of image acquisition devices, including an advanced kernel camera and its corresponding lighting system. A tongue image sample obtained using our device is shown in Fig. 2(a). Clearly, before the process of tongue classification, an exact region of the tongue must be extracted from an image, which almost always includes lips, part of the face or the teeth. Due to the fragmentary weakness of the tongue's edge and the pathological details on the surface of the tongue, however, traditional low-level image processing techniques, such as region growing and general edge detection, fail to segment the tongue from its surroundings, as shown in Fig. 2(b). The reason for this failure is that those methods do not take the shape property into account, which is an essential feature in such a task.

A model-based segmentation scheme, used in concert with image preprocessing, can overcome many of these limitations, 


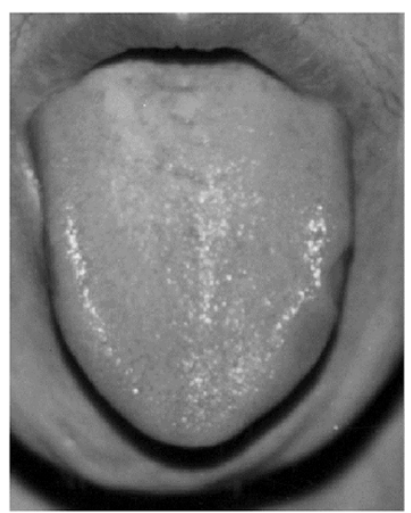

(a)

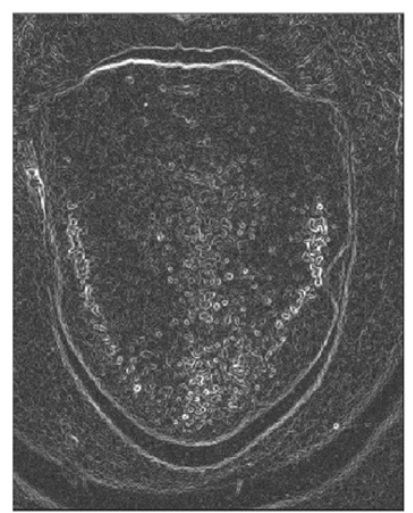

(b)
Fig. 2. A tongue image sample in our database and the image-enhancing result. (a) A tongue image from a patient suffering pancreatitis, and (b) its enhanced result using Sobel operator.

but such a scheme is not a panacea. Due to shape deformation and variation within object classes, the use of rigid templates, a typical model-based method, cannot produce satisfactory results in general. This realization has led to the use of deformable shape models in image segmentation.

Among model-based techniques, deformable models [18] offer a unique and powerful approach to image analysis that combines geometry, physics and approximation theory. They exploit (bottom-up) constraints derived from the image data together with (top-down) a priori knowledge about the location, size and shape of these structures. Deformable models are capable of accommodating the significant variability of biological structures over time and across different individuals.

Active contour models (ACM) or Snakes [1], as one of the most important classes of deformable shape models, are a sophisticated method for contour extraction and image interpretation. They advocate the view that the presence of an edge depends not only on the gradient at a specific point, but also on the spatial distribution. Snakes incorporate this global view of edge detection by assessing continuity and curvature combined with the local strength of an edge. The principal advantage of this approach over other feature extraction techniques is the integration of image data, an initial estimate of the contour, the desired contour properties and knowledge-based constraints in a single extraction process.

Although powerful and effective, the original ACM techniques have their intrinsic limits. Firstly, as a method tailored to semi-automatic applications, the initial contour of the ACM must, in general, be close to the true boundary or it will likely converge to an incorrect result. Secondly, during deformation, two undesirable effects may occur: shrinking of the contours of closed models due to the internal forces, and clustering or gathering of vertices in the corners of a model due to the external forces. Thirdly, in the presence of noise, ACM will find a lot of spurious edges; in other words, the flexibility of the active contours becomes a drawback. Finally, ACM uses only the information from its local surroundings, and the information about the global shape is missing. Several methods [2]-[6], [23]-[26] have been proposed to address these problems, but they can compromise other aspects of performance.
The deformable template (DT) [7]-[10], [16]-[22] matching scheme, on the other hand, provides an appealing solution to the segmentation tasks because of its capability to model an overall shape while accommodating shape variations. These deformable templates are specified by a set of parameters that enable a priori knowledge about the expected shape of the features to guide the detection process. The templates are flexible enough to be able to change their size, position and orientation, so as to match themselves to the image data. The inclusion of object-specific knowledge in a model enables the template-based method to be much more robust to occlusion and noise with respect to local deformable models, such as snakes. However, these global templates can neither exercise local control along the contour nor represent local distortion, which results in the loss of possibly meaningful details, such as tooth-marks along the rim of the body of the tongue that are used in TCM applications.

Deformable models based on superquadrics [27], [28] are another example of deformable shape template that can deform both locally and globally by incorporating the global shape parameters of a superellipsoid with the local degrees of freedom of a membrane spline in a Lagrangian dynamics formulation. The model's (six) global deformation degrees of freedom capture gross shape features from visual data, while the local deformation parameters reconstruct the details of complex shapes that the global abstraction misses. As a general-purpose model, however, the deformable superquadrics seems too symmetric to represent tongue shapes that usually take on a shape of "wider upside (the root of the tongue) and narrower downside (the tip of the tongue)".

Cootes et al. [16] presented a statistically based technique for building deformable shape templates and used these models to segment various organs from medical images. The statistical parameterization provides global shape constraints and allows the model to deform only in ways implied by the training set. The shape models represent objects by sets of landmark points which are placed manually in the same way on an object boundary in each input image. Whereas the idea of restricted elastic deformation of an average shape model is very promising, the parameterization of shapes by displacement of corresponding points on their contours is not a convenient technique, especially for large training sets. Staib and Duncan [29] cast the shape representation in the elliptic Fourier parameterization framework with probability distributions defined on the parameters of the representation, which biased the model toward an expected range of shapes. Boundary finding was formulated as an optimization problem using a maximum a posteriori objective function. Szekely et al. [30] combined the power of Fourier parameterization with statistical modal analysis. They propose Fourier snakes, which are represented by a parameter vector describing the mean contour and by a set of eigenmodes of the parameters characterizing the shape variation. An elastic fit of the mean model in the subspace of eigenmodes restricts possible deformations. Basically, the assumption underlying these approaches is that at some representational level, biological structures have a similar topology and differ only in shape details. It is, however, far from the case for tongue representation, since the tongue is a flexible 
organ that usually takes on greatly variability of shape over time and across different individuals. Moreover, the Fourier Snakes still requires manual displacement of albeit a small set of stable landmarks for each image under analysis, i.e., it is an interactive methodology.

In this paper, we combine DT with ACM to solve these problems. First, a deformable template consisting of a double ellipse (bielliptical model) is proposed to model a tongue, which gives a rough description of the shape of the tongue body. This model interacts with the edge map, which is a preprocessed result obtained by convoluting the source image with an image-enhancement filter, in a dynamic manner by changing its parameters to alter the position, orientation, size and other properties until the minimum of a predefined energy function is reached. Energy minimization is accomplished directly in the space of the parameters that are used to describe the bielliptical model. Then, the contour of the deformed model is properly sampled to obtain the best initial snake (from the fully automatic initialization point of view), called the Bielliptical Deformable Contour (BEDC). Different from the conventional snakes, the BEDC uses a brand-new term, called the template force, to replace the internal force. The main advantages of using the template force are: 1) it can drive the model to deform locally to represent the details while maintaining a consideration for the global shape, and 2) the template force, by its nature, provides an effective solution to undesirable deformation effects of traditional snakes, like shrinking and clustering. Finally, the BEDC can interpret tongue images in a totally automatic manner, which is essential to the automatic tongue diagnosis system.

\section{Bi-EllipticAl DeFormaBle TEMPlate FOR THE TONGUE}

\section{A. Definitions and Notations}

In geometry, an ellipse is defined as a curve in a plane, which is composed of all points that have an equal total distance from two fixed points, termed the focuses (or foci). From this point of view, a circle can be viewed as a specific case of an ellipse with two superposed focuses. Therefore, an ellipse can be intuitively viewed as a circle that has been inflated or compressed by a ratio of $b / r$ or $a / r$, respectively, along the horizontal or vertical axis, as shown in Fig. 3. This relationship can be directly derived from the regular equation of an ellipse as follows:

$$
x^{2} / a^{2}+y^{2} / b^{2}=1 \text {. }
$$

Then, multiplying both sides by $a^{2}$, we get

$$
x^{2}+\left(\frac{y}{b / a}\right)^{2}=a^{2}
$$

which can be viewed as the equation of a circle in an uneven space that is being stretched or contracted vertically (i.e., $y$-axis direction) by $b / a$. Thus, for each ellipse there is a corresponding circle, called a root circle, from which the ellipse originates. From this perspective, a root circle can be used to derive a sequence of ellipses by changing the ratio of compression or inflation along its two axes. Hereinafter, we use capital characters,

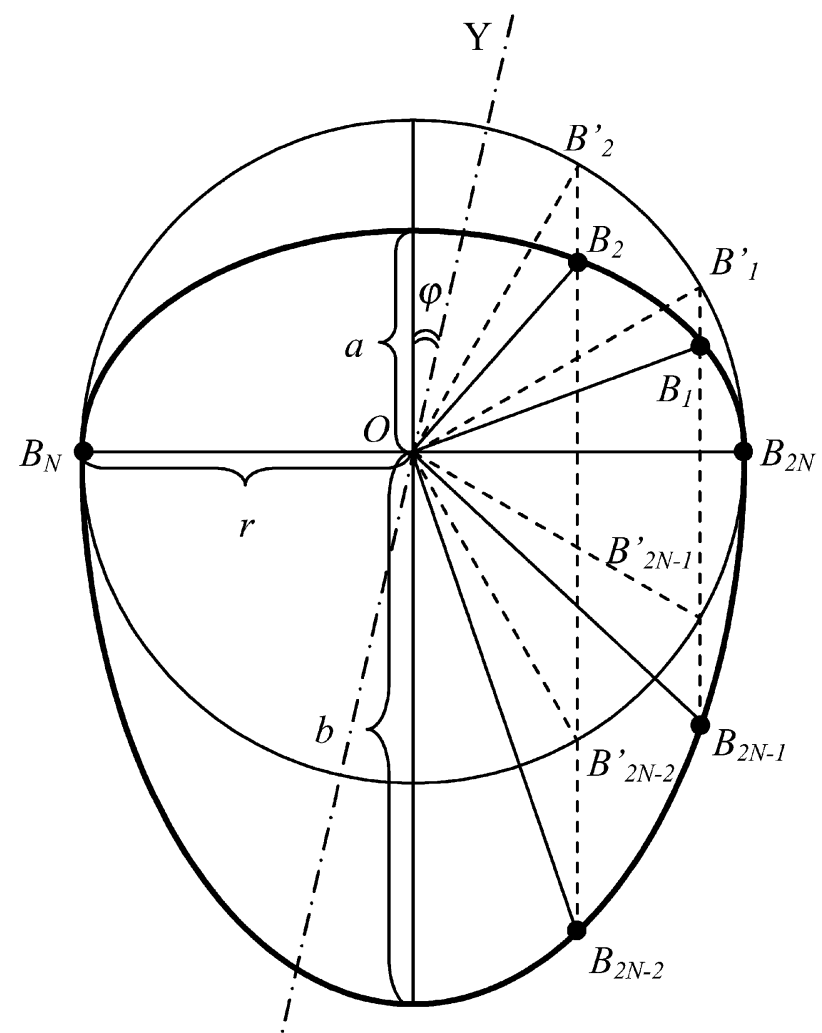

Fig. 3. Bi-elliptical tongue template (BEDT, drawn with a bold line) interpreted from the geometry point of view; and its root circle (with a thin line). $B_{i}, B_{i}^{\prime}(i=1,2, \ldots, 2 N)$ indicate nodes sampled on the boundary of the BEDT and their corresponding points on the root circle.

such as " $A$ " and " $B$," to denote nodes on an elliptical segment, and marked capital characters, such as " $A^{\prime \prime}$ " and " $B$ '," to denote points on the corresponding root circle.

\section{B. The Tongue Template}

Making use of the above-mentioned description of an ellipse, we present a specific deformable template for the tongue body, called Bi-Elliptical Deformable Template (BEDT, drawn with a bold line in Fig. 3). It is composed of two elliptical segments - the upper semi-ellipse and lower semi-ellipse, corresponding to the upper and lower boundary of the tongue body, respectively. Both of these segments have an identical root circle, namely circle, $O$, shown in Fig. 3, but they have different ratios: $a / r$ for the upper arc and $b / r$ for the lower arc. Depending on the introduction of the root circle, the six parameters used to describe the template are defined as follows:

- The coordinates of the template center, $\left(c_{x}, c_{y}\right)$, as the center of its root circle, namely point $O$.

- The length of the template radius, $r$, as the radius of its root circle.

- The ratios, $\left(\gamma_{a}, \gamma_{b}\right)$, as measurements of the lengths of the two vertical axes to $r$.

- The angle, $\varphi$, as the template orientations that are represented. (For a better representation of the template, we use an oblique vertical axis of the coordinate system in Fig. 3.) 
Using these parameters, each point, $\vec{x}(x, y)$, on the top arc of the template can be represented as

$$
\left[\begin{array}{l}
x \\
y
\end{array}\right]=\left[\begin{array}{l}
c_{x} \\
c_{y}
\end{array}\right]+\operatorname{rot}(\varphi) \cdot\left[\begin{array}{c}
r \cdot \cos \theta \\
r \cdot \sin \theta \cdot \gamma_{a}
\end{array}\right] \quad 0 \leq \theta \leq \pi
$$

and each point, $\vec{x}\left(x^{\prime}, y^{\prime}\right)$, on the bottom boundary of the template can be represented as

$$
\left[\begin{array}{l}
x^{\prime} \\
y^{\prime}
\end{array}\right]=\left[\begin{array}{l}
c_{x} \\
c_{y}
\end{array}\right]+\operatorname{rot}(\varphi) \cdot\left[\begin{array}{c}
r \cdot \cos \theta \\
r \cdot \sin \theta \cdot \gamma_{b}
\end{array}\right] \quad \pi \leq \theta \leq 2 \pi
$$

where $\theta$ is the angle between $\vec{x}$ and the positive direction of the horizontal axis, and $\operatorname{rot}(\varphi)$ is the rotation matrix

$$
\operatorname{rot}(\varphi)=\left[\begin{array}{cc}
\cos \varphi, & -\sin \varphi \\
\sin \varphi, & \cos \varphi
\end{array}\right]
$$

The six parameters are placed together in a vector, $\mathbf{p}=$ $\left(c_{x}, c_{y}, r, \gamma_{a}, \gamma_{b}, \varphi\right)$, which can be updated over time by minimizing an energy function that is defined later.

\section{Energy Function for the Tongue Template}

We now define the potential energy for the BEDT, which is a function of its parameter vector. The minimum of the energy function corresponds to the best fit with the interested features, namely the edges in our application. The parameters of the template can be updated by some rules. This corresponds to following a certain path in the parameter space of the model.

Different from the one used with a snake, such an energyminimizing framework has the positive effect of constraining the motion of every single point on a curve, in each iteration, to the motion of all the other points. The direct consequence is an improvement in the global behavior of the model, where the motion of every single point on a curve is only linked to that of adjacent points and to the local forces arising from the image.

Generally, the energy functions of a model can be divided into two parts: the internal energy and the external energy. The internal energy function stores a priori information about the feature to be extracted, which will take no effect here since the deformation is performed in the parameter space. The external energy function is chosen so that it has its minima on the high contrast regions in the image. Because edges consist of points with high contrast values, the energy will be minimal on those points. Given a gray-level image, $I(x, y)$, the external energy function of the tongue template is given by the following relationship:

$$
\begin{aligned}
& E_{\text {ext }}=\frac{1}{\text { Length }} \int_{\text {Bi-elliptical-curve }} P(\vec{x}) d s \\
& P(\vec{x})=-|\nabla I(x, y)| \text { or } \\
& P(\vec{x})=-\left|\nabla\left(G_{\sigma} * I(x, y)\right)\right|
\end{aligned}
$$

where $G_{\sigma}$ is a two-dimensional Gaussian function with standard deviation $\sigma, \nabla$ is the gradient operator, and $P(\vec{x})$ is the external force field. It is easy to see from these definitions that larger values of $\sigma$ will cause the boundaries to become blurrier. Such large $\sigma$ values are often necessary, however, to increase the capturable range of the template and reduce noise as well.
The external energy term can also be written as a function of the parameter values (regardless of the orientation parameter) as follows:

$$
\begin{aligned}
E_{\text {ext }}= & \frac{1}{L_{a}} \int_{\text {Upper-elliptic-curve }} P\left(c_{x}+r \cdot \cos \theta, c_{y}\right. \\
& \left.+r \cdot \sin \theta \cdot \gamma_{a}\right) d s+\frac{1}{L_{b}} \int_{\text {Lower-elliptic-curve }} P\left(c_{x}\right. \\
& \left.+r \cdot \cos \theta, c_{y}+r \cdot \sin \theta \cdot \gamma_{b}\right) d s
\end{aligned}
$$

where $L_{a}$ and $L_{b}$ correspond to the arc length of the upper and lower elliptical curves, respectively. Note that scale independence is achieved by dividing the curvilinear integrals by the arc length.

The parameters of the deformable template are computed over time by minimizing the energy function, $E_{\text {ext }}$, by applying the Euler-Lagrange equations. In other words, the minimization is done by using the steepest descent method on the energy function in the parameter space. Thus, the update rule of the parameter vector, $\mathbf{p}$, is given by

$$
\frac{d \mathbf{p}}{d t}+\frac{\partial E_{\mathrm{ext}}}{\partial \mathbf{p}}=0
$$

To set up an iterative solution, let the curve be sampled on the variable, $\theta$, as follows:

$$
\theta \Rightarrow \theta_{i}=2 \pi \cdot i / 2 N .
$$

It should be noted that $\theta$ is the sample step on the root circle of the template. Then, the external energy computed on the set of nodes $\left(B_{i}\right.$, shown in Fig. 3$)$ can be written as

$$
E_{\mathrm{ext}}=\frac{1}{2 N} \sum_{i=1}^{2 N} P\left(x_{i}, y_{i}\right) .
$$

Similarly, (7) can be rewritten as follows:

$$
\begin{aligned}
E_{\mathrm{ext}} & =\frac{1}{N} \sum_{i=1}^{N} P\left(c_{x}+r \cdot \cos \theta_{i}, c_{y}+r \cdot \sin \theta_{i} \cdot \gamma_{a}\right) \\
& +\frac{1}{N} \sum_{i=N+1}^{2 N} P\left(c_{x}+r \cdot \cos \theta_{i}, c_{y}+r \cdot \sin \theta_{i} \cdot \gamma_{b}\right) .
\end{aligned}
$$

Equation (8) evaluated on the set of nodes is

$$
\begin{aligned}
\frac{d \mathbf{p}}{d t} & =-\frac{\partial E_{\text {ext }}}{\partial \mathbf{p}}=-\frac{\partial E_{\text {ext }}}{\partial \mathbf{x}} \cdot \frac{\partial \mathbf{x}}{\partial \mathbf{p}} \\
& =-\left(\frac{\partial E_{\text {ext }}}{\partial x} \cdot \frac{\partial x}{\partial \mathbf{p}}+\frac{\partial E_{\text {ext }}}{\partial y} \cdot \frac{\partial y}{\partial \mathbf{p}}\right) \\
& =-\frac{1}{2 N} \sum_{i=1}^{2 N}\left(\frac{\partial P\left(x_{i}, y_{i}\right)}{\partial x} \cdot \frac{\partial x}{\partial \mathbf{p}}+\frac{\partial P\left(x_{i}, y_{i}\right)}{\partial y} \cdot \frac{\partial y}{\partial \mathbf{p}}\right)
\end{aligned}
$$

where $\mathbf{x}=(x, y)$.

To compute the $\mathbf{p}$ time derivatives and the partial derivatives of $P(x, y)$ along the curve, let the index $n$ correspond to $t$, and 
the time step for each iteration be $\Delta t$. Then, the required partial derivatives can be approximated as

$$
\begin{aligned}
\mathbf{p}_{t} & =\frac{1}{\Delta t}\left(\mathbf{p}^{n+1}-\mathbf{p}^{n}\right) \\
\frac{\partial P(x, y)}{\partial x} & =\frac{P(x+1, y)-P(x-1, y)}{2} \\
\frac{\partial P(x, y)}{\partial y} & =\frac{P(x, y+1)-P(x, y-1)}{2} .
\end{aligned}
$$

The derivatives $\partial x / \partial \mathbf{p}$ and $\partial y / \partial \mathbf{p}$ can be obtained from (3) and (4), respectively. Finally, these equations give our iterative solution to the BEDT.

The iteration should be stopped when an energy minimum is reached. However, this could lead to the algorithm stopping prematurely due to a local minimum in the energy function, which is caused by noisy data. This problem can be overcome by allowing the algorithm to continue even though the value of the energy function is not decreasing. The stopping criteria may involve interrupting the algorithm if a lower value for the energy function is not found within a certain number of iterations. Simulated annealing [11] and dynamic programming [12] have also been proposed to reduce the problems caused by local minima. In practice, BEDTs always work well by using the steepest decent method for the minimization of their energy functions, we thus do not employ these methods in this paper.

\section{Combined Model for Tongue Segmentation}

Although powerful and robust, the BEDT, as a global DT, has certain intrinsic limitations. For example, it is difficult to represent local details. Locally deformable models such as snakes, which are capable of performing local control along the track of the features, can be used to solve this problem. However, active contour models or snakes usually suffer difficulties in initialization and global shape representation. To address these problems, we intuitively combine the two models. First, we use the BEDT to obtain a rough description of the contour of the tongue body. After the minimum of the BEDT energy function is reached, the resultant contour is then re-sampled to obtain the best initialization for a subsequent snake (in a fully automated manner). To incorporate global shape constraints and shape information in the model, and to remove the unwanted contraction and clustering forces while retaining the local continuity and smoothness constraints, we introduce an original term called the template force, which replaces the conventional internal forces. Then, the dynamic contour model actively modifies its shape, under the influences of both the template force field and an external force field derived from some image feature energy distribution, until it enters a stationary state at the end.

\section{A. Two Kinds of Template Forces}

A traditional snake is a curve, $v(s)=[x(s), y(s)], s \in[0,1]$, which moves through the spatial domain of an image to minimize the energy function, that is

$$
E=\int_{0}^{1}\left(E_{\text {int }}(v(s))+E_{\text {ext }}(v(s))\right) d s
$$

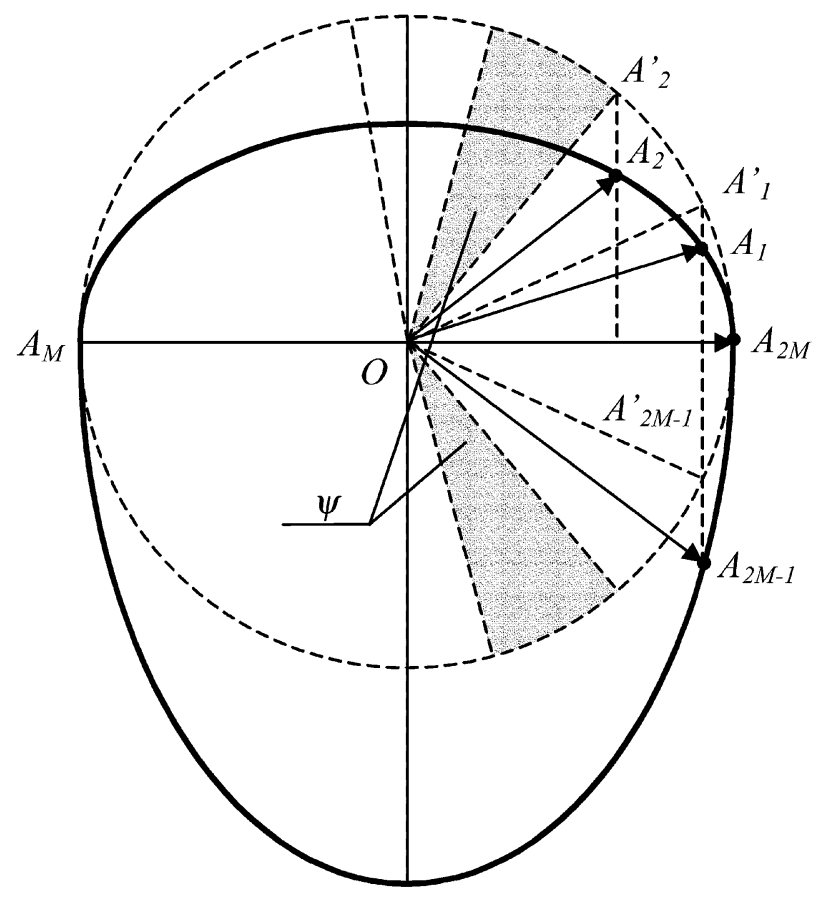

Fig. 4. Sampling rule for the combined model. A tongue template and its root circle are drawn in bold and dashed line, respectively (Sampling interval, $\psi$, is a constant angle on the root circle). $A_{i}, A_{i}^{\prime}(i=1,2, \ldots, 2 M)$ denote nodes on the boundary of the BEDT and corresponding points on the root circle.

where $E_{\text {int }}(v(s))$ and $E_{\text {ext }}(v(s))$ are the internal and external energy functions, respectively, that can be formulated as

$$
\begin{aligned}
& E_{\text {int }}(v(s))=\int_{0}^{1}\left(\alpha\left|v^{\prime}(s)\right|^{2}+\beta\left|v^{\prime \prime}(s)\right|^{2}\right) d s \\
& E_{\text {ext }}(v(s))=\gamma \int_{0}^{1} P(v(s)) d s
\end{aligned}
$$

where $\alpha$ and $\beta$ are weighting parameters that control the snake's tension and rigidity, respectively, $P(v(s))=P(x, y)=$ $-\left|\nabla\left[G_{\sigma} * I(x, y)\right]\right|$ denotes a scalar potential function defined on the image plane $I(x, y)$, and $\gamma$ is a weighting parameter that controls the magnitude of the potential. It can be seen from (14) that when $\left|E_{\text {ext }}(v(s))\right|$ is small, the energy is dominated by the internal term, that is, the weighted sum of the squares of the first and second derivatives of $v(s)$. This is the origin of the snake's inherent contraction force.

To address this problem, we first sample the resultant contour of the BEDT on the variable, $\psi$, to obtain a set of nodes, $A_{i}(\mathrm{i}=$ $1,2, \ldots, 2 \mathrm{M})$. It should be noted that $\psi$ is the sample step on the root circle of the tongue template (drawn with a dashed line in Fig. 4). Then, let all of the nodes be forced to move radially. That is, when driven by external and template forces, each node, $A_{i}$, will only change its position bi-directionally along the line, $\mathrm{OA}_{i}$, instead of moving unconstrainedly in the force fields as the snake allow [1]. Obviously, such a deformation constraint will remove the tangential shrinking force caused by the traditional internal forces.

To provide a definition for the template forces, we present a concept called "radius" for each node along the contour. 
Similar to the template radius defined in Section II-B, the radius of a node is given by

$$
R_{i}=x_{i} / \cos (i \cdot \psi)
$$

where $x_{i}$ is the x-coordinate of node, $A_{i}$. It can be seen from (16) that all radiuses of the nodes are initially identical to the template radius, $r$, and they will take on various values as the contour modifies its shape. To describe the average dimensions of the deformable contour, we introduce a variable, $R_{\mathrm{avg}}$, as follows:

$$
R_{\mathrm{avg}}=\frac{1}{2 M} \sum_{i=1}^{2 M} R_{i} .
$$

1) Linear Template Force (LTF): Similar to the rigidity term in the internal energy function in (15), the linear template energy can be written as follows:

$$
E_{\mathrm{tmp}}^{(1)}=\frac{1}{2 M} \sum_{i=1}^{2 M}\left(1+\frac{\overrightarrow{A_{i} A_{i-1}} \cdot \overrightarrow{A_{i} A_{i+1}}}{\left|\overrightarrow{A_{i} A_{i-1}}\right| \cdot\left|\overrightarrow{A_{i} A_{i+1}}\right|}\right) .
$$

We wish to stress here that, in a global sense, a linear template force will exhibit concave potentials when applied to the energy minimization of a closed convex contour, thus it is also called the concave template force. Even with that restriction, a linear template force is extremely useful in many applications since it can exercise unbiased local control along the contour, which is a significant characteristic in the absence of a priori information about the shape.

The linear template force, $\hat{F}_{\mathrm{tmp}-l, i}$, which is illustrated in Fig. 5(a) as $F_{i}$, is therefore formulated as

$$
\hat{F}_{\mathrm{tmp}-l, i}=c_{i}\left|\overrightarrow{\mathrm{OA}_{i+1}}+\overrightarrow{\mathrm{OA}_{i-1}}-2 \overrightarrow{\mathrm{OA}_{i}}\right| \hat{e}_{i}
$$

where $c_{i}>0$ is the weighting parameter and $\hat{e}_{i}$ is the unit vector in the direction of $\overrightarrow{\mathrm{OA}_{i}}$, as shown in Fig. 5(a).

2) Elliptical Template Force (ETF): The elliptical template energy is calculated by making use of a relationship between the length of the radiuses of each node and its adjacent neighbors, as follows:

$$
E_{\mathrm{tmp}}^{(2)}=\frac{1}{2 M} \sum_{i=1}^{2 M}\left|\frac{R_{i-1}+R_{i+1}}{2}-R_{i}\right| .
$$

The corresponding elliptical template force, $\hat{F}_{\mathrm{tmp}-e, i}$, shown in Fig. 5(b) as $F_{i}$, can be written as

$$
\hat{F}_{\mathrm{tmp}-e, i}=d_{i}\left|R_{i+1}+R_{i-1}-2 R_{i}\right| \hat{e}_{i}
$$

where $d_{i}>0$ is the weighting coefficient, $R_{i}$ is the radius [referring to (16)] and $\hat{e}_{i}$ is the unit vector in the direction of $\overrightarrow{O A_{i}^{\prime}}$, as shown in Fig. 5(b). Note that in (18)-(21), " -" and "+" in the subscripts are both operators modulo- $2 M$, and the factor $1 / 2 M$ is added to make the template energies independent of the number of sampled nodes along the contour.

Unlike the linear template force, the elliptical template force will drive the contour to take on a regular shape where the external force field is weak or zero. Furthermore, the final shape of

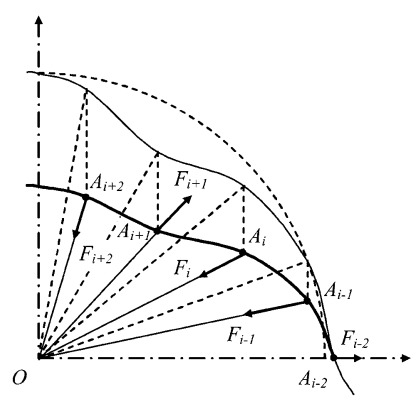

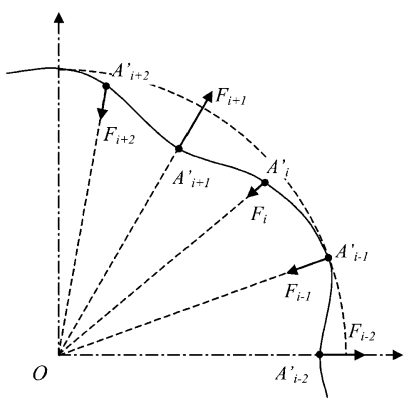

(b)
Fig. 5. Two kinds of the template force for the BEDC. (a) Linear template force and (b) Elliptical template force (an initial root circle is in dashed line, an intermediate figure of the deformed root circle and the corresponding contour segment in thin and bold line, respectively). Note that, the contour segment of the BEDC is not drawn in (b) since elliptical template forces directly act on nodes along the root circle.

the active contour in such a force field can be the BEDT itself provided there is no image data anywhere. Note that the way the elliptical template force acts on a node is indirect; in other words, it modifies the position of a node by changing the length of the radius of that node. A very important characteristic of the ETF is that, when it is combined with template constraints, the locally defined formulation can represent the global shape and impose local control simultaneously. Therefore, the elliptical template energy could have a special advantage if information about the shape is available.

\section{B. Bi-Elliptical Deformable Contours}

We introduce a new snake, called the Bi-Elliptical Deformable Contours (BEDC), by replacing the internal energy term with the template energy functions defined in the previous section. Here, the new active contour is defined as a set of massless nodes and is driven by both template and external forces that originate from the template and external potential energies, respectively. Shape constraints and information are incorporated into the term for the template forces. The discrete energy function of the BEDC can be written as

$$
\begin{aligned}
E^{(1)}= & \frac{1}{2 M} \sum_{i=1}^{2 M}\left(w_{\text {ext }} P\left(x_{i}, y_{i}\right)\right. \\
& \left.+w_{\text {tmp }}\left(1+\frac{\overrightarrow{A_{i} A_{i-1}} \cdot \overrightarrow{A_{i} A_{i+1}}}{\left|\overrightarrow{A_{i} A_{i-1}}\right| \cdot\left|\overrightarrow{A_{i} A_{i+1}}\right|}\right)\right)
\end{aligned}
$$

or

$$
\begin{aligned}
E^{(2)}= & \frac{1}{2 M} \sum_{i=1}^{2 M}\left(w_{\text {ext }} P\left(x_{i}, y_{i}\right)\right. \\
& \left.+w_{\text {tmp }}\left|\frac{R_{i-1}+R_{i+1}}{2}-R_{i}\right|\right)
\end{aligned}
$$

where $2 M$ denotes the number of nodes sampled along the contour and $P\left(x_{i}, y_{i}\right)$ is the external force field defined in (6). The weighting factors, $w_{\text {ext }}$ and $w_{\mathrm{tmp}}$, control the tradeoff between the external and template forces and may have default values for each application, but they allow modification by the operator. By emphasizing the external forces, one will make the model follow 
the extracted image features more precisely, whereas placing emphasis on the template forces will smooth out the path of the contour.

To eliminate the problem of shrinking in traditional snakes, we will make some modifications to the term for the external forces. In fact, vertex displacement along the path of the contour model does not make any contribution to the deformation of the model and it is even a defect. Therefore, we will not use the external force $\left(\hat{F}_{\text {ext }}\right)$ itself, but instead decompose it into its local radial and tangential components, and we only use the local radial component to drive the vertices of the contour model. If we denote the local radial component of $\hat{F}_{\text {ext }, i}$ by $\hat{F}_{\text {ext }, r_{i}}$, it can be calculated as a dot product by

$$
\hat{F}_{\text {ext }, r_{i}}=\left(\hat{F}_{\text {ext }, i} \cdot \hat{e}_{i}\right) \hat{e}_{i}
$$

where $\hat{e}_{i}$ is the unit vector in the local radial direction of the ellipse [for the linear template model in Fig. 5(a)] or the root circle [for the elliptical template model in Fig. 5(b)]. The local radial forces, $\hat{F}_{\mathrm{ext}, r_{i}}$ and $\hat{F}_{\mathrm{tmp}, i}\left(\hat{F}_{\mathrm{tmp}-l, i}\right.$, or $\left.\hat{F}_{\mathrm{tmp}-e, i}\right)$, provide a resulting driving force on a vertex of the contour model that is purely devoted to deformation of the model without moving the vertex along the path of the contour.

As a result of the forces that act on a vertex, this vertex will start to move and change its position until it enters an equilibrium position. Such a process will be performed for each node along the contour in a single iteration. When all nodes come to a rest position in the force field, the resultant contour gives the final solution. The actual deformation procedure is implemented as a so-called numerical time integration process in which the complete state of the contour model is calculated at a sequence of discrete positions in time.

\section{Tongue Segmentation Algorithm}

The overall approach for tongue segmentation is composed of two main procedures: minimization of the energy functions of the BEDT and BEDC, as illustrated in Fig. 6. Three main points should be emphasized here. Firstly, the calculations of both energy functions are based on a predefined edge map, $h(x, y)$, that is derived from a gray-level channel, $I(x, y)$, of a true color tongue image, which has the property that it is larger near the edges of the image. To make the external energy function take on its smallest values at the boundaries, we define the external force field as

$$
P(x, y)=-h(x, y) .
$$

Secondly, the external force fields for the BEDT and BEDC are actually different since they are calculated based on different levels in a scale space. It is the dissimilar purposes of the BEDT and BEDC that lead to such a difference. In fact, the BEDT is applied to roughly locate the boundary starting from an arbitrary initial position, which might be far away from the true edges, in an edge map full of noise and fragmentary weakness. Therefore, for the BEDT, emphasis should be placed on the robustness and the relatively large capturable range. Accordingly, larger values of $\sigma$ for edge-enhancing operators, such as the Laplacian of Gaussian operation, are usually used to compute the edge map, so as to remove the noise and enlarge the capturable range of the

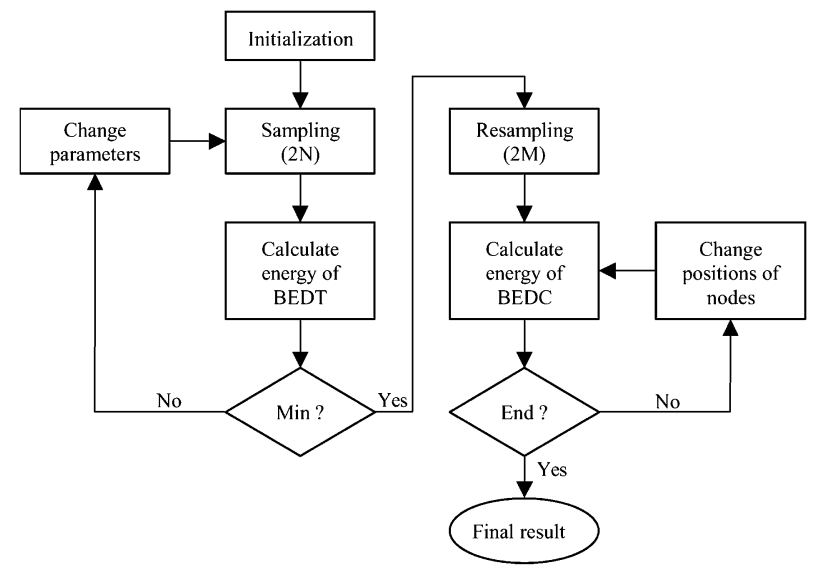

Fig. 6. Flowchart of tongue segmentation procedure using the BEDC.

model. In contrast, the BEDC is used to refine the intermediate resultant contour, given by the BEDT, within a relatively small distance near the true boundary. In this case, a smaller value of $\sigma$ is often employed to obtain better local control.

Finally, the sampling intervals in the BEDT and BEDC can also be different, and they are usually evaluated so that $M>N$. The reason for this is similar to that stated above: the intention of the BEDT process, performed in the parameter space, is to roughly locate the boundary. Thus, a reasonable small number of sampling points will be enough; furthermore, it can significantly reduce the computation time. Whereas the BEDC process is used to represent local features, consequently a relatively large number of sampling nodes could be more helpful.

\section{EXPERIMENTAL RESULTS AND ANALYSIS}

We have developed a prototype implementation of the described approach and applied the BEDC to a total of 3572 samples that were purposely chosen (for diagnosis) from our database, which contains over 12000 clinical tongue images. In this section, we will present some useful results.

In all of the following experiments, we use the red channel of the original tongue image as the test sample, which is actually a gray-level image, so as to highlight the tongue domain because red is usually the dominant color within tongue body images. To compute the energy functions, we first need to calculate the edge map. The two possibilities are $h^{(1)}(x, y)=|\nabla I(x, y)|$ and $h^{(2)}(x, y)=\left|\nabla\left(G_{\sigma}(x, y) * I(x, y)\right)\right|$, where the latter is more robust in the presence of noise and it can enlarge the capturable range of the models. Moreover, Gaussian filters with a time-varying standard deviation can be used to further improve the performance with respect to both stability and accuracy [14]. Other more complicated noise-removal techniques, such as median filtering and morphological filtering, could also be applied to improve the underlying edge map. For all of the experiments, we used $w_{\text {ext }}=1.0$ and $w_{\mathrm{tmp}}=0.8$.

The first example is illustrated in Fig. 7, which shows the results of applying the BEDC as well as original snakes to a real tongue image $(640 \times 480$ pixels, each 24 bits). Because of the limited capturable range of the traditional snakes, the initial contours [two connected parabolic segments shown with black 


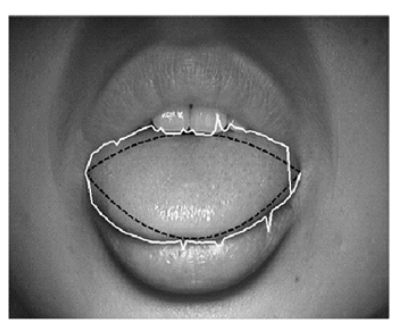

(a)

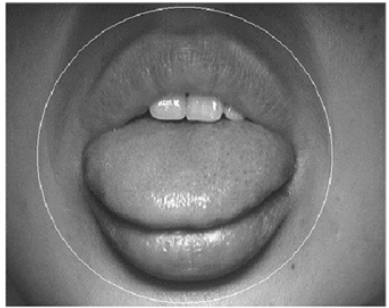

(c)

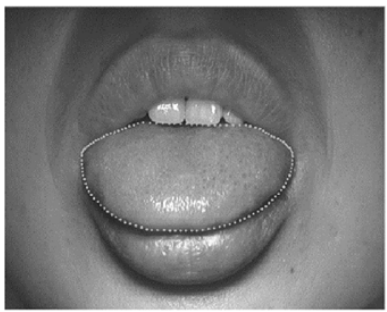

(e)

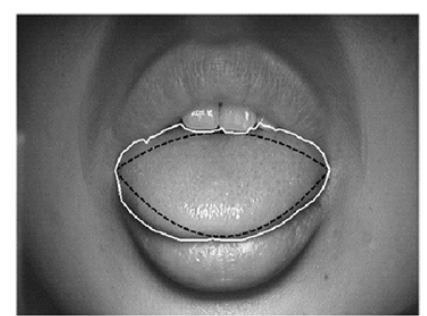

(b)

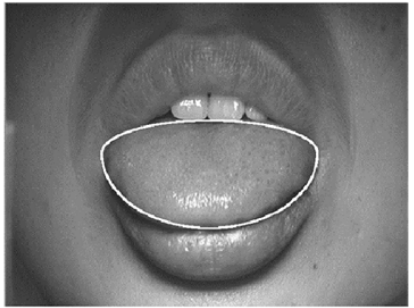

(d)

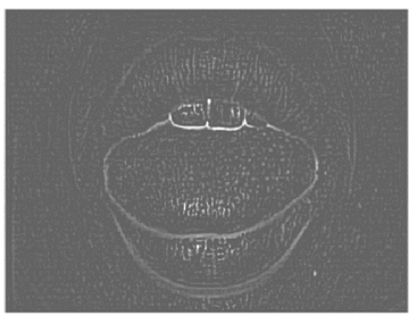

(f)

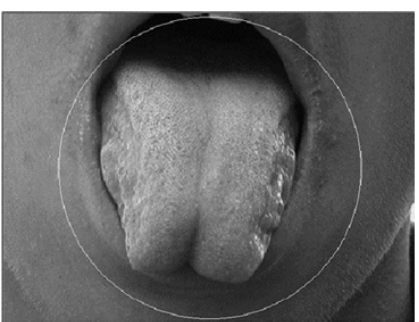

(a)

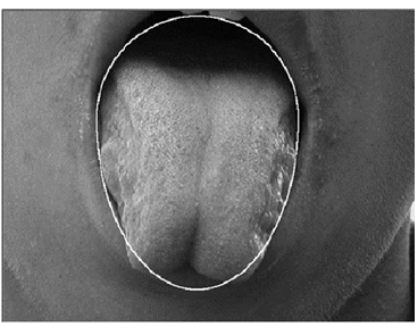

(c)

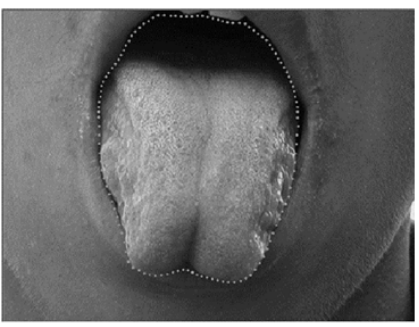

(e)

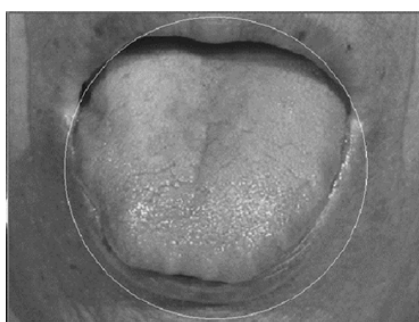

(b)

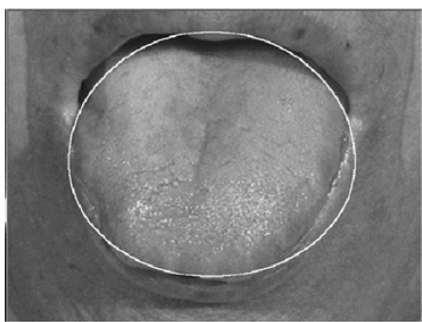

(d)

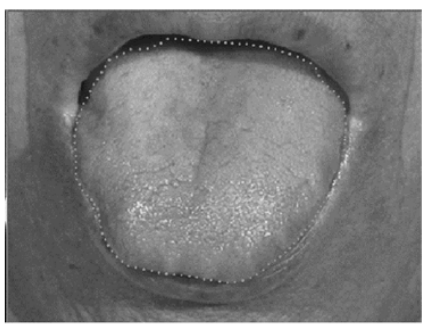

(f)

Fig. 7. Comparison between a Snake and the BEDC. Edge extraction using a traditional snake with (a) $\beta=0.2$ and (b) $\beta=0.8$. (c) An initial contour for the BEDT. (d) The resulting template of the BEDT. (e) The final resulting contour using the BEDC. (f) An edge map, $\left|\nabla\left(G_{\sigma} * I\right)\right|$, with $\sigma=3$.

dashed lines in Fig. 7(a)-(b)] are placed near the real edge of the tongue body. Here, we make a modification to both snakes; the parameters are evaluated as $\alpha \gg \beta, \gamma$ [see (15)], which are the weighting parameters of the internal and external terms, respectively [1], so as to remove the shrinking force. It can be seen from Fig. 7(a) and (b) that, in both cases the traditional snake failed to locate the real edges due to their flexibility and sensitivity to noises (pay attention to the upper resulting contour near teeth in both figures; and the first snake with a smaller beta value was entrapped into several spurious edges caused by reflecting points). The reason for the failure of a traditional snake is that there are lots of pathological details and glistening points on the tongue's surface. These details have turned into noise points when filtered by the edge-enhancing templates, as shown in Fig. 7(f). In contrast, the initial contour for the BEDT can be set far away from the true contour, as illustrated in Fig. 7(c), since the search approach of the BEDT can provide a large capturable range. Thanks to its global control as the energy minimization progresses as it acts like a DT and its robustness to noise, the BEDT can give a rather good representation to the boundary of the tongue, as shown in Fig. 7(d), without becoming entrapped in spurious edges. Finally, Fig. 7(e) shows the resulting contour by using the BEDC that performs a posterior local refinement based on the resultant model. It is clear that the BEDC exhibits better performance than traditional snakes in dealing with noises.

Fig. 8. Comparison between the DT and BEDC. (a) An initial curve on a captured image of a tongue with a long, deep vertical crack in the midline reaching to the tip. (b) An initial curve on a captured image of a partial swollen tongue on the half edge. (c)-(d) The intermediate contours obtained by using the DT. (e)-(f) The final contours by applying the BEDC.

Fig. 8 illustrates the BEDC's capability for local control as compared with the DT method. In this example, the BEDT is first utilized to model the tongue body. Different from any local models, such as snakes, deformable templates incorporate global shape information into their parameter vectors, and thus they show better stability in the presence of noise. However, they are usually only able to give a rough description of the features and they encounter difficulties in exact local search, as shown in Fig. 8(c) and (d), which means they are far from meeting the requirements for tongue diagnosis in Chinese medicine. The reasons can be explained as follows. First, tongues of different people always present a variety of different shapes as a result of dissimilar physiological structures (referring to the examples in this paper). Even a series of captured sample images of the tongue from the same person can take on different shapes since the tongue is a movable apparatus. Therefore, no single predefined DT can model all of the possible shapes of the tongue bodies. Second, the examination of the shape of the tongue body is one of the most important aspects of tongue diagnosis, where the physical contour of the tongue plays a significant role. Although many medical disorders generally appear as a variation in the shape of the tongue body, (such as thin or swollen, long or short, etc.) local distortions along the contour of the tongue, such as tooth marks and partial 


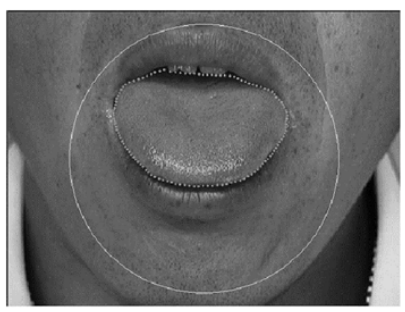

(a)

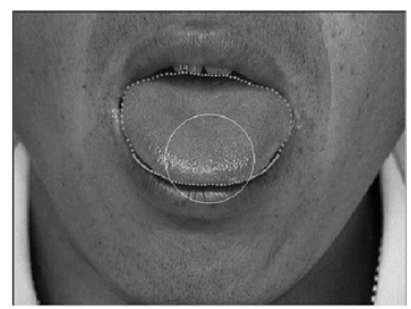

(c)

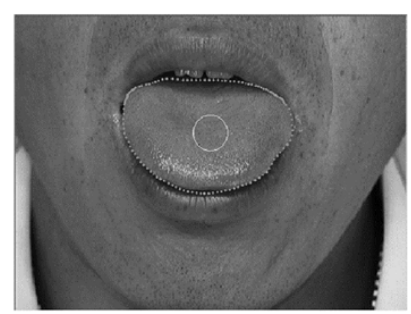

(b)

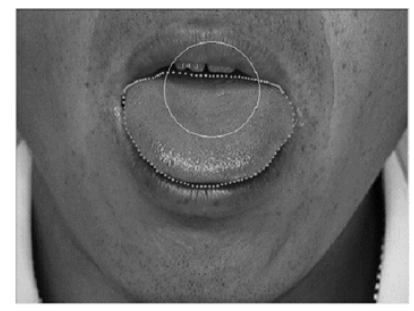

(d)
Fig. 9. Two significant improvements of the BEDC: insensitivity to the initial location of the contour and having a large capturable range. Convergence of the BEDC starting from different initial positions: (a) outside the tongue's boundary, (b) inside the tongue's boundary, and (c), (d) overlapping with the upper and lower edges of the tongue, respectively.

swelling [see Fig. 8(b)], are usually more meaningful hints for diagnosis.

Fig. 8(a) and (b) presents two typical tongue bodies. One tongue has a long, deep vertical crack in the midline reaching to the tip, while the other is partially swollen on the right edge and is accompanied by light tooth marks. The resulting contours of these tongues are shown in Fig. 8(e) and (f) using a combined model that is composed of a BEDT and its derived BEDC. Both of the results clearly demonstrate the effective local control of our approach.

We next discuss the sensitivity of the final result to the shape of the initial contour. In other words, how reproducible is the result when we start from different initial contours? The following example demonstrates two significant advantages of the BEDC: insensitivity to the initial position of the contour and a large capturable range. Fig. 9 shows the different initial contours (as solid pale lines) that were used in our experiments: 1) far outside the tongue's boundary [Fig. 9(a)]; 2) inside the tongue's boundary [Fig. 9(b)]; and 3) overlapping with the upper and lower edges of the tongue [Fig. 9(c) and (d), respectively]. In all cases, the traditional snake stops in a very undesirable configuration due to its limited capturable range and its poor robustness to noise (although the results are not shown here). In the model developed by Cohen [2], the potential forces are directly imposed on a snake, thus it may allow some partial segments of the contour to become trapped in spurious edges due to its flexibility. However, the BEDC utilizes a DT to search for the location of the tongue's boundary in the external potential force field so that a much better representation, from a global point of view, is obtained. It can be seen that the results do not depend strongly on the location of the initial contour, which is actually one of the reasons for using a DT model instead of drawing the contours manually. In a number of well-defined applications, it seems feasible to generate the initial contour without any user

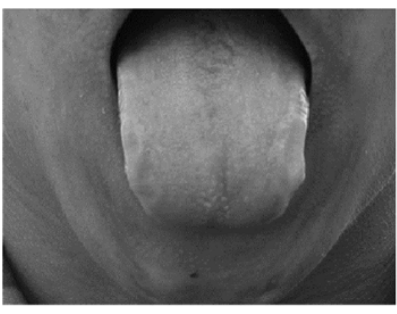

(a)

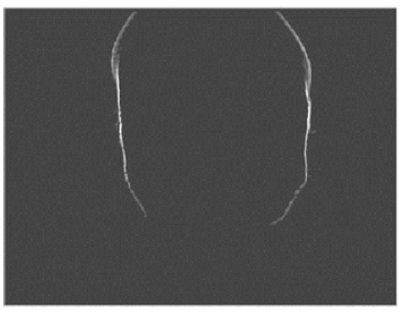

(c)

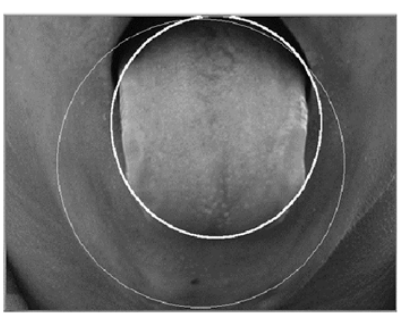

(e)

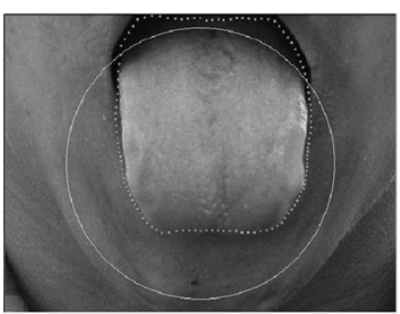

(g)

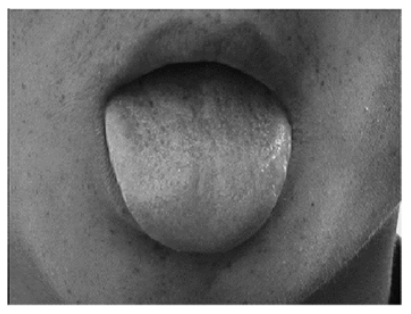

(b)

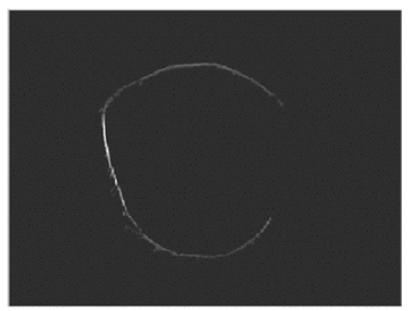

(d)

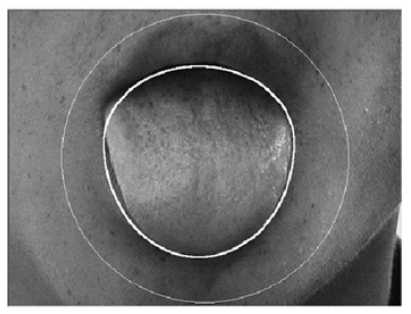

(f)

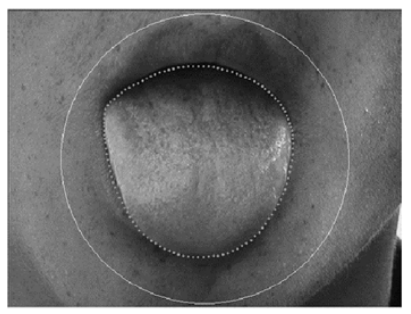

(h)
Fig. 10. Two examples showing the particular effectiveness of the template force of the BEDC. (a)-(b) Two original clinical images; (c)-(d) Over-filtered edge maps using a LoG with $\sigma=5$ and $\sigma=3$, respectively; (e)-(f) The resulting contours (shown with bold solid line) by using the BEDT starting from a initial contour (drawn with thin solid line); and (g)-(h) The final resulting contours by using the BEDC with an elliptical template force.

interaction, thereby making the entire approach automatic and reproducible.

The last clinical example is presented in Fig. 10, where our attention now turns to the particular effectiveness of the template forces of the BEDC. It is found universally that there are many pathological features on the surface of the tongue, such as points, spots and thorns or prickles. When filtered by certain edge-enhancing operators, these features may be transformed to such strong edges that they can even overwhelm the information about the tongue's boundary. This obviously adds difficulties to the problem of tongue segmentation and common edge detection methods become helpless in such a situation. One possible solution is to over-filter the original images, such as by applying a Laplacian of Gaussian operator with large $\sigma$, to smooth out the troublesome pathological features. However, such an approach only solves one problem while it creates another: the boundary of the tongue body 
exhibits fragmentary weakness in the over-filtered edge map, as shown in Fig. 10(c)-(d). Nevertheless, the BEDC model can address this problem thanks to the template forces creating the potentials that make the model take on a specific shape.

Fig. 10(a)-(b) shows the original images whose over-filtered edge maps are created by applying a Laplacian of Gaussian filter with $\sigma=5$ and $\sigma=3$, respectively [see Fig. 10(c)-(d)]. It can be seen that the resultant boundaries become fragmentary weak, and they are even blank on the upper and lower edges of the tongue for Fig. 10(c) and on the right edges for Fig. 10(d). The resulting models of the BEDT are displayed in Fig. 10(e)-(f) with bold solid lines, which apparently give a coarse representation of the boundaries of the tongue bodies. The final results, depicted in Fig. 10(g)-(h), reveal that, by substituting the elliptical template force for both the tensile and rigid internal forces, the BEDC would rather take on a regular shape (elliptical curves in this case) and it provides a good estimation for the real edges in the regions with zero potential forces, rather than contracting together as a conventional snake usually does. The reason for such a characteristic in the BEDC is that the elliptical template force, as a whole, can exercise global control due to the root circle, although it has a local formulation.

We have applied the BEDC to a total of 3572 clinical tongue images, out of which 3405 samples (about $95.3 \%$ of the total number) are successfully segmented. Note that, all the experimental samples are gray level images (red color plane of true color bitmaps), therefore we do not assess the performance of our algorithm quantitatively. Instead, we use qualitative indices "success" and "failure", where "success" indicates a satisfactory result for the follow-up feature analysis process in the automatic tongue diagnosis system (see Fig. 1) and "failure" otherwise.

This impressive degree of success of the algorithm stems from its robustness to noises and sparse data, and the ability to conduct the local and global controls in a consistent way. Generally, deformable models experience difficulties in dealing with noisy data, in other words, the flexibility becomes a drawback. Solutions to this problem include the incorporation of shape constraints, exploiting global information, or the use of multiscale techniques. These methods, however, usually employ separate local and global controls, resulting in the incapability of dealing with sparse data. The main reason for this kind of weakness is the complete ignorance of the global shape representation when the model is conducting the local deformation. Therefore, the model can seldom make valid assumption of the missing contour segments via local control. The BEDC, on the contrary, can overcome this limitation by applying the template force, which can drive the contour take on regular shapes according to current global representation in the absence of edge datapoints. Finally, the insensitivity of the BEDC to initial position makes the initialization step totally unnecessary (we can use fixed parameters for all samples), which exactly fills the need for automatic tongue segmentation.

In some cases, however, the BEDC fails to find the correct tongue contour (about $4.7 \%$ of the 3572 samples get wrong results). Fig. 11 shows a typical example of unsuccessful segmentation. Analysis of the results has shown that the tongue and its

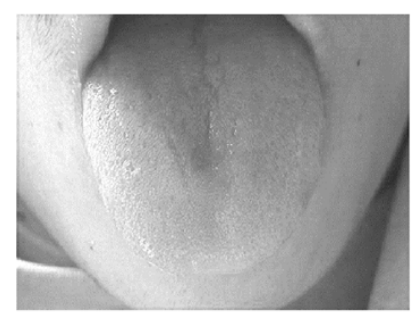

(a)

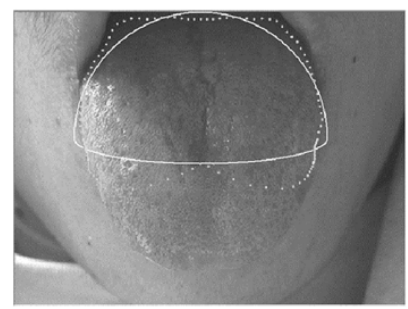

(c)

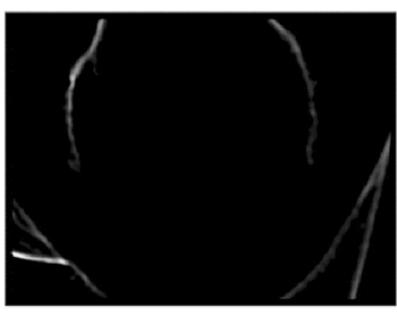

(b)
Fig. 11. Typical example illustrating segmentation failure of the BEDC. (a) Gray-level image showing the red color plane of a tongue image, (b) the corresponding edge map, and (c) the resultant BEDT (in bold solid line) and BEDC.

surroundings may occasionally take on similar values on the red color plane, especially around the tip of the tongue (see Fig. 11). Therein, the edges become very vague or even totally missing, which is mainly responsible for the failure. A potential solution is to develop color-enhancing techniques rendering the tongues prominent from their surroundings.

\section{CONCLUSION}

In this paper, we develop a new deformable contour approach, the BEDC, for automated tongue edge extraction in the tongue diagnosis system. Based on a combination of the BEDT and ACM, our proposed approach can exercise both local and global controls in a consistent way by introducing a template force, originating from the BEDT, into the energy function of the BEDC to replace the traditional internal force terms. Experimental results from 3572 clinical tongue images demonstrate that our new approach is able to interpret tongue images in a fully automatic manner due to the insensitivity to initial positions and robustness to noises and sparse data. Moreover, the main idea in the BEDC, namely realizing a global shape representation only through local control, might also be useful in other edge detection applications provided a specific template force could be suitably defined.

\section{ACKNOWLEDGMENT}

The authors would like to thank B. Huang, M. He, and F. Li for providing aids in the experiments.

\section{REFERENCES}

[1] M. Kass, A. Witkin, and D. Terzopoulos, "Snakes: Active contour models," in Proc. Int. Conf. Computer Vision, 1987, pp. 259-269.

[2] L. D. Cohen, "On active contour models and balloons," CVGIP: Image Understand., vol. 53, no. 2, pp. 211-218, Mar. 1991.

[3] T. McInerney and D. Terzopoulos, "Topologically adaptable snakes," in Proc. Int. Conf. Computer Vision, 1995, pp. 840-845. 
[4] K. F. Lai and R. T. Chin, "On regularization, formulation and initialization of the active contour models (snakes)," in Proc. Asian Conf. Computer Vision, 1993, pp. 542-545.

[5] S. R. Gunn and M. S. Nixon, "A robust snake implementation: A dual active contour," IEEE Trans. Pattern Anal. Machine Intell., vol. 19, no. 1, pp. 63-68, Jan. 1997.

[6] C. Y. Xu and J. L. Prince, "Snakes, shapes, and gradient vector flow," IEEE Trans. Image Processing, no. 3, pp. 359-369, Mar. 1998.

[7] A. L. Yuille, P. W. Hallinan, and D. S. Cohen, "Feature extraction from faces using deformable templates," in Int. J.Comput. Vis., vol. 8, 1992, pp. 99-111.

[8] A. Jain, Y. Zhong, and S. Lakshmanan, "Object matching using deformable templates," IEEE Trans. Pattern Anal. Machine Intell., vol. 18, no. 3, pp. 267-278, Mar. 1996.

[9] K. Mardia, W. Qian, D. Shah, and K. Desouza, "Deformable template recognition of multiple occluded objects," IEEE Trans. Pattern Anal. Machine Intell., vol. 19, no. 9, pp. 1035-1042, Sep. 1997.

[10] D. Terzopoulos, "On matching deformable models to images: Direct and iterative solutions," in OSA Tech. Dig., vol. 2, 1987, pp. 160-167.

[11] G. Storvik, "A Bayesian approach to dynamic contours through stochastic sampling and simulated annealing," IEEE Trans. Pattern Anal. Machine Intell., vol. 16, no. 10, pp. 976-986, Oct. 1994.

[12] D. Gelger, A. Gupta, L. A. Costa, and J. Vlontzos, "Dynamical programming for detecting, tracking and matching deformable contours," IEEE Trans. Pattern Anal. Machine Intell., vol. 17, no. 3, pp. 294-302, Mar. 1995.

[13] G. Maciocia, Tongue Diagnosis in Chinese Medicine. Seattle, WA: Eastland, 1995.

[14] B. Pang, K. Q. Wang, and D. Zhang, "Time-adaptive snakes for tongue segmentation," in Proc. Int. Conf. IG, vol. 5, 2000, pp. 228-331.

[15] D. Zhang, Automated Biometrics: Technologies and Systems. Norwell, MA: Kluwer, 2000.

[16] T. F. Cootes, C. J. Taylor, D. H. Cooper, and J. Graham, "Active shape models-Their training and application," Comput. Vis. Image Understand., vol. 61, no. 1, pp. 38-59, Jan. 1995.

[17] G. Hamarneh and T. Gustavsson, "Combining snakes and active shape models for segmenting the human left ventricle in echocardiographic images," Comput. Cardiol., pp. 115-118, 2000.

[18] T. McInerney and D. Terzopoulos, "Deformable models in medical image analysis: A survey," Med. Image Anal., vol. 1, no. 2, pp. 91-108, 1996.
[19] F. L. Valverde, N. Guil, J. Munoz, Q. Li, M. Aoyama, and K. Doi, "A deformable model for image segmentation in noisy medical images," in Proc. Int. Conf. Image Processing, vol. 2, 2001, pp. 82-85.

[20] S. Joshi, S. Pizer, P. T. Fletcher, P. Yushkevich, A. Thall, and J. S. Marron, "Multiscale deformable model segmentation and statistical shape analysis using medial descriptions," IEEE Trans. Med. Imag., vol. 21, no. 5, pp. 538-550, May 2002.

[21] C. Nikou, G. Bueno, F. Heitz, and J. P. Armspach, “A joint physics-based statistical deformable model for multimodal brain image analysis," IEEE Trans. Med. Imag., vol. 20, no. 10, pp. 1026-1037, Oct. 2001.

[22] A. Scaggiante, R. Frezza, and M. Zampato, "Identifying and tracking ellipses: A technique based on elliptical deformable templates," in Proc. ICIAP, 1999, pp. 582-587.

[23] B. R. Lee, A. B. Hamza, and H. Krim, "An active contour model for image segmentation: A variational perspective," in IEEE Int. Conf. Acoustics, Speech, Signal Processing, vol. 2, 2002, pp. 1585-1588.

[24] W. N. Lie and C. C. Chuang, "Fast and accurate snake model for object contour detection," Electron. Lett., vol. 37, no. 10, pp. 624-626, May 2001.

[25] H. W. Park, T. Schoepflin, and Y. Kim, "Active contour model with gradient directional information: Directional snake," IEEE Trans. Circuits Syst. Video Technol., vol. 11, no. 2, pp. 252-256, Feb. 2001.

[26] V. Caselles, R. Kimmel, and G. Sapiro, "Geodesic active contours," in Proc. Int. Conf. Computer Vision, Cambridge, MA, 1995, pp. 694-699.

[27] D. Terzopoulos and D. Metaxas, "Dynamic 3D models with local and global deformations: Deformable superquadrics," IEEE Trans. Pattern Anal. Machine Intell., vol. 13, no. 7, pp. 703-714, Jul. 1991.

[28] D. Metaxas and D. Terzopoulos, "Shape and nonrigid motion estimation through physics-based synthesis," IEEE Trans. Pattern Anal. Machine Intell., vol. 15, no. 6, pp. 580-591, Jun. 1993.

[29] L. H. Staib and J. S. Duncan, "Boundary finding with parametrically deformable models," IEEE Trans. Pattern Anal. Machine Intell., vol. 14, no. 11, Nov. 1992.

[30] G. Szekely, A. Kelemen, C. Brechbuhler, and G. Gerig, "Segmentation of 2-D and 3-D objects from MRI volume data using constrained elastic deformations of flexible Fourier contour and surface models," Med. Image Anal., vol. 1, no. 1, pp. 19-34, 1996. 